\title{
Esaki Diodes in van der Waals Heterojunctions with Broken-Gap Energy Band Alignment
}

Rusen Yan, ${ }^{\dagger}{ }^{\dagger}$ Sara Fathipour, ${ }^{\ddagger}$ Yimo Han,, Bo Song, ${ }^{\dagger \dagger}$ Shudong Xiao, ${ }^{\dagger}$ Mingda Li, $^{\dagger}$ Nan Ma, ${ }^{\dagger}$ Vladimir Protasenko, ${ }^{\ddagger}$ David A. Muller, ${ }^{\|, \perp}$ Debdeep Jena, ${ }^{\dagger, \ddagger, \S}$ and Huili Grace Xing ${ }^{*}, \dagger, \downarrow, \S$

${ }^{\dagger}$ School of Electrical and Computer Engineering, Cornell University, Ithaca, New York 14583, United States

${ }^{\ddagger}$ Department of Electrical Engineering, University of Notre Dame, Notre Dame, Indiana 46556, United States

${ }^{\S}$ Department of Materials Science and Engineering, Cornell University, Ithaca, New York 14583, United States

"School of Applied and Engineering Physics, Cornell University, Ithaca, New York 14853, United States

${ }^{\perp}$ Kavli Institute at Cornell for Nanoscale Science, Ithaca, New York 14853, United States

Supporting Information

ABSTRACT: van der Waals (vdW) heterojunctions composed of twodimensional (2D) layered materials are emerging as a solid-state materials family that exhibits novel physics phenomena that can power a range of electronic and photonic applications. Here, we present the first demonstration of an important building block in vdW solids: room temperature Esaki tunnel diodes. The Esaki diodes were realized in vdW heterostructures made of black phosphorus (BP) and tin diselenide $\left(\mathrm{SnSe}_{2}\right)$, two layered semiconductors that possess a broken-gap energy band offset. The presence of a thin insulating barrier between BP and $\mathrm{SnSe}_{2}$ enabled the observation of a prominent negative differential resistance (NDR) region in the forward-bias current-voltage characteristics, with a peak to valley ratio of 1.8 at $300 \mathrm{~K}$ and 2.8 at $80 \mathrm{~K}$. A weak temperature dependence of the NDR indicates electron tunneling being
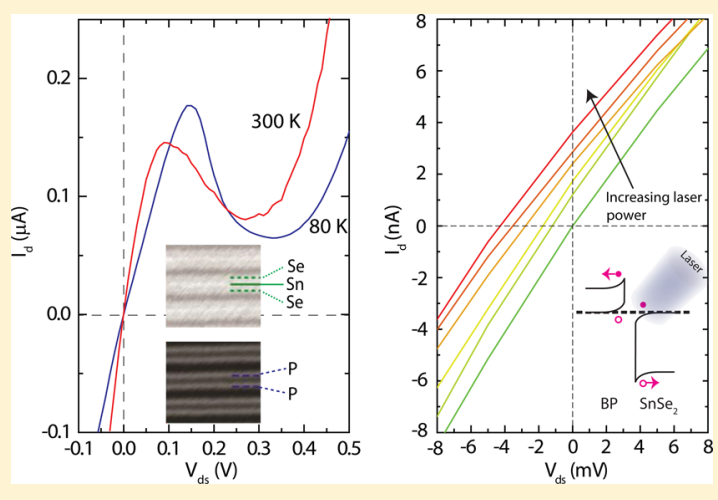
the dominant transport mechanism, and a theoretical model shows excellent agreement with the experimental results. Furthermore, the broken-gap band alignment is confirmed by the junction photoresponse, and the phosphorus double planes in a single layer of BP are resolved in transmission electron microscopy (TEM) for the first time. Our results represent a significant advance in the fundamental understanding of vdW heterojunctions and broaden the potential applications of 2D layered materials.

KEYWORDS: van der Waals heterojunction, Esaki diode, tunneling junction, negative differential resistance (NDR), black phosphorus (BP), tin diselenide $\left(\mathrm{SnSe}_{2}\right)$

$\mathrm{E}$ saki's discovery of negative differential resistance (NDR) in heavily doped semiconducting germanium $p-n$ junctions in 1958 was the first experimental evidence of quantum mechanical tunneling transport of electrons in allcondensed-matter systems. ${ }^{1,2}$ This discovery motivated Giaever's tunneling experiments that proved the existence of the superconductive energy gap predicted by the then-newly formulated Bardeen-Cooper-Schrieffer (BCS) theory of superconductivity. ${ }^{3}$ After these initial breakthroughs, tunneling in various classes of crystalline matter has been observed and forms the basis for several practical applications. For example, Josephson junctions exploit tunneling in superconductors for exquisitely sensitive magnetic flux detectors in superconducting quantum interference devices (SQUIDs) ${ }^{4}$ and are now being investigated as the building blocks of quantum computers. ${ }^{5}$ Electron tunneling forms the basis for low-resistance ohmic contacts to heavily doped semiconductors for energy-efficient transistors, as low-loss cascade elements in multijunction solar cells, and for coherent emission of long-wavelength photons in

quantum-cascade lasers. $^{6,7}$ In addition to such practical applications, the extreme sensitivity of tunneling currents to various electronic, vibrational, and photonic excitations of solids makes tunneling spectroscopy one of the most sensitive probes for such phenomena. ${ }^{8}$

Recently, interband tunneling in semiconductors has been proposed as the enabler for a new class of semiconductor transistors called tunnel field-effect transistors (TFETs) that promise very low-power operation. The heart of such devices is an Esaki tunnel diode, with preferably a near broken-gap band alignment at the source-channel heterojunction. ${ }^{9,10}$ As these heterojunction TFETs are scaled down to the nanometer regime, the increase in bandgap barrier due to quantum confinement may significantly prohibit the desired tunneling currents because tunneling current decreases exponentially with

Received: May 6, 2015

Revised: July 7, 2015

Published: July 30, 2015 

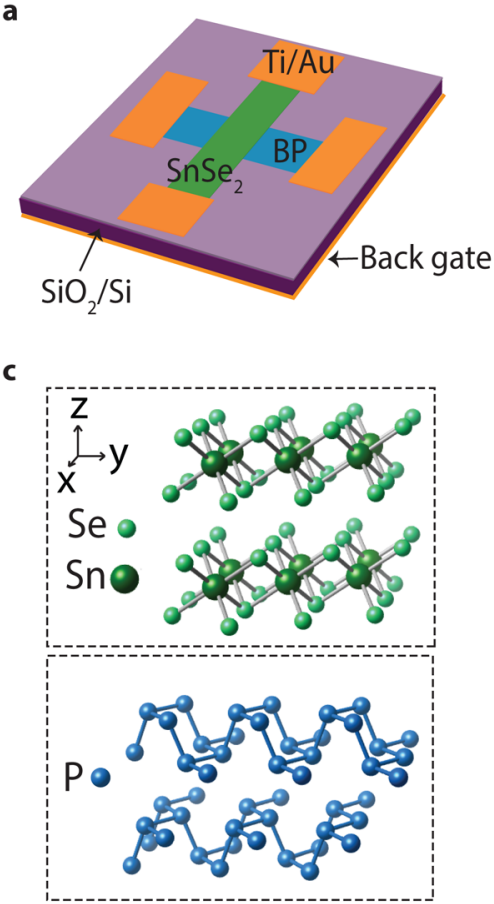

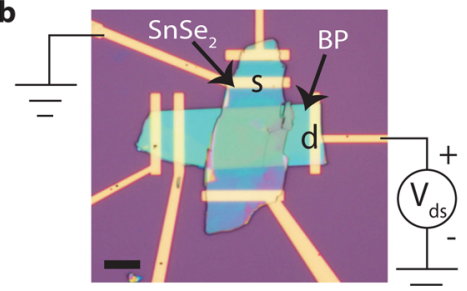

d

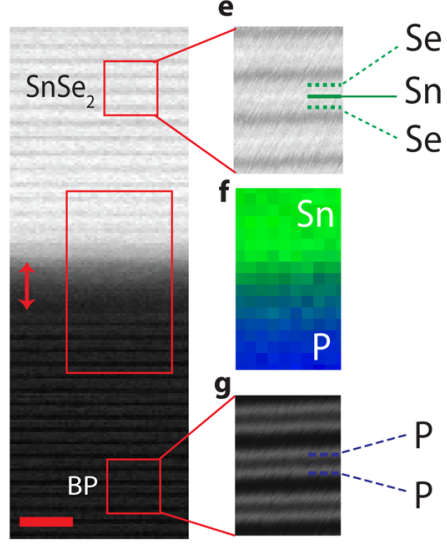

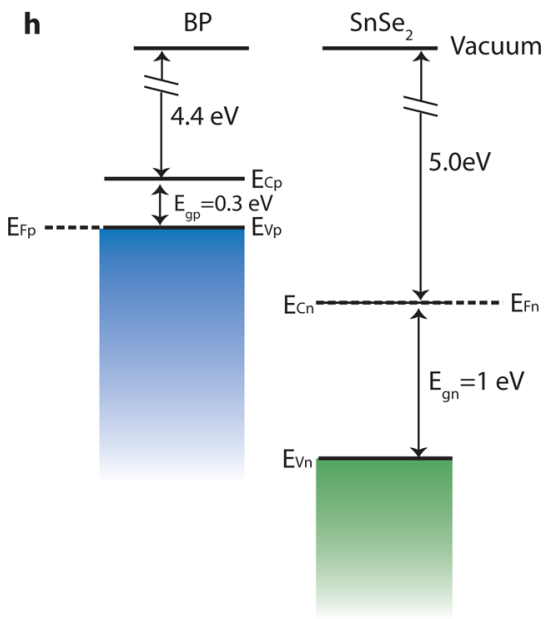

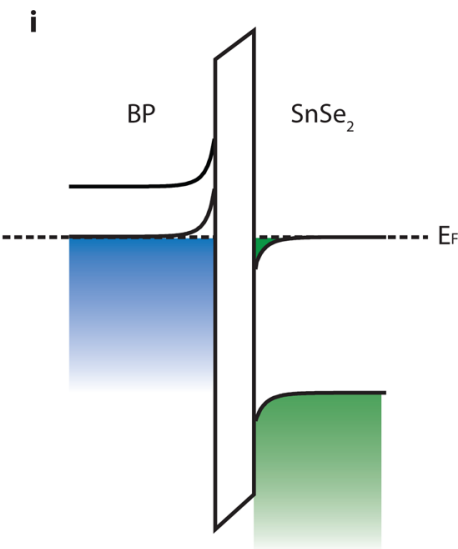

Figure 1. Structure and energy band alignment of the $\mathrm{BP} / \mathrm{SnSe}_{2}$ vdW heterojunction. $(\mathrm{a}, \mathrm{b})$ Schematic illustration and optical image of fabricated devices on $\mathrm{SiO}_{2} / \mathrm{Si}$ substrates. The scale bar is $5 \mu \mathrm{m}$. A voltage $V_{\mathrm{ds}}$ is applied on the p-type BP with the n-type SnSe $e_{2}$ grounded. (c) Crystal structures of $\mathrm{BP}$ and $\mathrm{SnSe}_{2}$. BP is composed of two planes of phosphorus atoms arranged in puckered layers, and $\mathrm{SnSe}_{2}$ has a CdI type structure with each plane of $\mathrm{Sn}$ atoms sandwiched between two planes of Se atoms. Layers in $\mathrm{BP}$ or $\mathrm{SnSe}_{2}$ are bonded together by vdW force, which allows exfoliation of these crystals into thin flakes. (d,e,g) Cross-sectional STEM images: (d) shows the presence of a thin barrier $(\sim 1.6 \mathrm{~nm})$ at the interface. The scale bar is $2 \mathrm{~nm}$; (e) shows the triatomic-plane layer structure of $\mathrm{SnSe}_{2} ;(\mathrm{g})$ shows the biatomic-plane layer structure of BP. The measured interlayer distance of $\mathrm{SnSe}_{2}$ is $0.65 \mathrm{~nm}$ and that of BP is $0.55 \mathrm{~nm}$. (f) EELS map: $\mathrm{Sn}$ in green and P in blue. (h) Energy band profiles of $\mathrm{BP}$ and $\mathrm{SnSe} \mathrm{e}_{2}$ prior to contacting each other. ${ }^{23,24} \mathrm{BP}$ has a low work function compared to that of $\mathrm{SnSe}_{2}$. Due to unintentional doping, exfoliated BP and $\mathrm{SnSe} \mathrm{e}_{2}$ flakes onto $\mathrm{SiO}_{2} / \mathrm{Si}$ substrates are p-type ${ }^{25}$ and heavily n-type doped, respectively (also see Supplementary Figure S1). (i) Band alignment at equilibrium. Accumulations of holes in $\mathrm{BP}$ and electrons in $\mathrm{SnSe}_{2}$ result from electron transfer from $\mathrm{BP}$ to $\mathrm{SnSe}_{2}$ owing to a lower work function of $\mathrm{BP}$ than that of $\mathrm{SnSe}_{2}$. The degenerately doped $\mathrm{p}-\mathrm{BP}$ and $\mathrm{n}-\mathrm{SnSe}_{2}$ along with the thin insulating barrier in between form the heart of the Esaki diode.

the barrier height. Layered semiconductors with a sizable bandgap and a wide range of band alignments can potentially avoid such degradation and have been proposed as ideally suited for such applications. ${ }^{11,12}$ This class of devices distinguishes themselves from the graphene-based SymFET by offering a desired low off-current. ${ }^{13,14}$ Compared to traditional 3D heterojunctions, such structures are expected to form high-quality heterointerfaces due to the absence of dangling bonds. ${ }^{1,15-17}$ The weak van der Waals (vdW) bonding in principle does not suffer from lattice mismatch requirements and makes strain-free integration possible. Among the previous reports on vdW solids, heterojunctions of type-I (straddling) and type-II (staggered) band alignments have been demonstrated. ${ }^{17-21}$ Very recently, Roy et al. reported an Esaki NDR at low temperatures in an as-stacked multilayer $\mathrm{MoS}_{2} / \mathrm{WSe}_{2}$ heterojunction, which is believed to possess a type-II band alignment, employing dual gates. ${ }^{22}$ However, the NDR observed by them did not persist to room temperature. In 
this work, two layered materials, black phosphorus (BP) and tin diselenide $\left(\mathrm{SnSe}_{2}\right)$, are successfully integrated for the first time, enabling the conclusive achievement of Esaki-diode behavior in two-dimensional (2D) crystal semiconductors at room temperature. We use the Esaki diodes to experimentally prove that the heterojunction possesses a type-III (broken-gap) band alignment. The $\mathrm{BP} / \mathrm{SnSe}_{2}$ heterojunction is one between mixedvalence materials because black phosphorus is elemental and $\mathrm{SnSe}_{2}$ is a compound semiconductor. Furthermore, the two constituents have different crystal structures. Given all these differences, it is rather remarkable that a robust NDR can be observed at room temperature in this heterostructure.

The Esaki diode device structure is schematically shown in Figure 1a. The devices are fabricated using a dry transfer process with flake thicknesses of $\sim 50-100 \mathrm{~nm}$ for both BP and $\mathrm{SnSe}_{2}{ }^{26} \mathrm{BP}$ is the p-type semiconductor, and $\mathrm{SnSe}_{2}$ the n-type semiconductor of the vdW Esaki diode. A detailed device fabrication and characterization procedure is provided in the Method section. Figure $1 \mathrm{~b}$ shows an optical image of a representative device. The thicknesses of $\mathrm{BP}$ and $\mathrm{SnSe}_{2}$ in this device are 79 and $95 \mathrm{~nm}$, determined by atomic force microscopy (AFM). Figure 1c shows the crystal structures of $\mathrm{BP}$ and $\mathrm{SnSe}_{2}$. BP, the most stable allotrope of phosphorus obtained under high pressure possesses an orthorhombic crystal structure. Each phosphorus atom is covalently bonded to three adjacent atoms in a puckered layer, thus forming two atomic planes in each layer of BP. ${ }^{27}$ In comparison, $\mathrm{SnSe}_{2}$ consists of planes of $\mathrm{Sn}$ sandwiched between two planes of Se atoms, forming a hexagonal $\mathrm{CdI}_{2}$-type structure. ${ }^{28}$ Similar to other $2 \mathrm{D}$ crystals, layers are held together by vdW forces in both BP and $\mathrm{SnSe}_{2}$. Figure 1d shows the high-angle annular dark-field scanning transmission electron microscopy (HAADF-STEM) images of the $\mathrm{SnSe}_{2}-\mathrm{BP}$ interface. Figure 1e,g are the zoomedin HAADF-STEM images of $\mathrm{SnSe}_{2}$ and BP, showing the triatomic-plane $\mathrm{SnSe}_{2}$ layers with an interlayer distance of 0.65 $\mathrm{nm}$ and the biatomic-plane BP layers with an interlayer separation of $0.55 \mathrm{~nm}$. The measured layer thicknesses for both materials are very close to the values in the literature. At the interface, an amorphous layer with a thickness ranging from 1.2 to $2 \mathrm{~nm}$ is observed, which is thicker than a typical vdW gap of 0.4-0.6 nm. ${ }^{22,29}$ Electron energy loss spectroscopy (EELS) composition analysis of this layer is shown in Figure $2 \mathrm{f}$. It reveals carbon and traces of phosphorus and $S n$, but no oxygen above the noise floor (see Supplementary Figure S3). We speculate that the presence of carbon most likely stems from the flake transfer process, while the presence of $\mathrm{P}$ and Sn likely results from the finite interdiffusion, assisted by the degradation of the BP and $\mathrm{SnSe}_{2}$ flake surfaces prior to being stacked together due to their widely known instability in air. ${ }^{30,31} \mathrm{We}$ can not completely rule out that $\mathrm{BP}$ and $\mathrm{SnSe}_{2}$ near the interface suffered from degradation during the TEM sample preparation and imaging process, as observed by other groups. ${ }^{29}$ However, it is worth highlighting that the double atomic planes in a BP layer is resolved by TEM for the first time in this work, to the best of our knowledge. This can be attributed to the aberration correction up to the fifth order and the low electron beam energy used: $100 \mathrm{keV}$. More details on STEM can be found in the Methods section.

High doping densities boost the NDR characteristics in traditional semiconductor Esaki diodes, which apply in these vdW Esaki diodes as well. The BP flake is unintentionally ptype doped, and the $\mathrm{SnSe}_{2}$ flake is unintentionally doped $\mathrm{n}$ type. This is consistent with other groups' observations. ${ }^{25,32} \mathrm{We}$
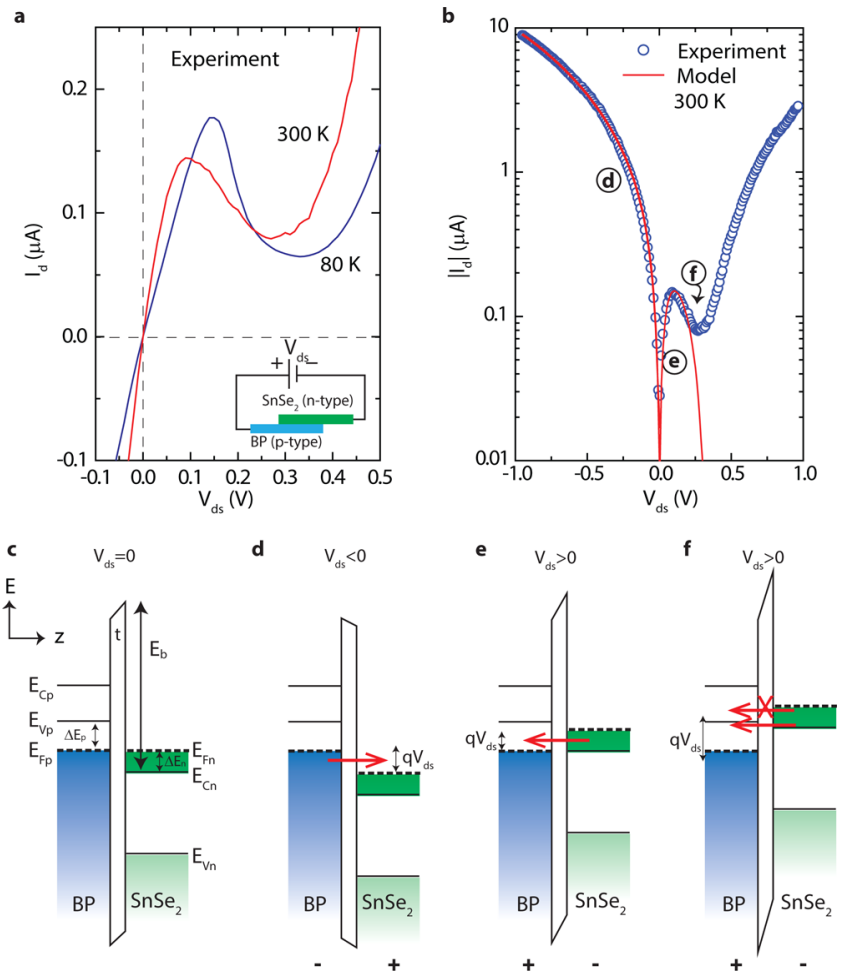

Figure 2. Current-voltage characteristics of the $\mathrm{BP} / \mathrm{SnSe}_{2}$ vdW Esaki tunnel diode. (a) $I_{d}-V_{d s}$ curves at 80 and $300 \mathrm{~K}$ in a linear scale. At lower temperatures, the peak current increases and the valley current decreases thus an increase in the peak-to-valley ratio, as expected from the theory and consistent with the prior reported Esaki diodes. ${ }^{4}$ (b) Comparison of experimental and theoretical $I_{d}-V_{d s}$ curves at $300 \mathrm{~K}$ in a log scale. Excellent agreement is achieved. (c-f) Band alignment at $V_{d s}=0, V_{d s}<0$, and $V_{d s}>0$. When the conduction band electrons in $\mathrm{SnSe}_{2}$ have a maximal overlap with the valence band holes in BP $\left(V_{d s}>\right.$ 0 ), the Esaki tunnel diode reaches its peak current. With further increasing $V_{d s}(\mathrm{f})$, part of the conduction band electrons in $\mathrm{SnSe}_{2}$ see the forbidden band gap of $\mathrm{BP}$, thus leading to a reduction in current.

confirm the individual doping type unambiguously by the opposite directions of field-effect conductivity modulation of each layer (Supplementary Figure S1a). That the effective doping densities are high manifests in the relatively weak current modulation in the individual flakes, which is also attributed to the large flake thicknesses. Doping in $2 \mathrm{D}$ materials is poorly understood and controlled in general today. Studies suggested that unintentional doping in $2 \mathrm{D}$ materials can be induced by defects, including vacancies and impurities, ${ }^{32-34}$ and adsorbates such as oxygen and $\mathrm{NO}_{2}{ }^{35}$ Similar to carbon nanotube FETs, ${ }^{36}$ one manifestation of the ambiguous doping in $2 \mathrm{D}$ crystals is variations in threshold voltage of the $2 \mathrm{D}$-crystal FETs. Indeed, variations are observed in the individual BP and $\mathrm{SnSe}_{2}$ FETs as well as the stacked heterostructures in this work.

Figure $1 \mathrm{~h}$ shows the energy level alignments of conduction and valence band edges of the $p$-type BP and $n$-type $\mathrm{SnSe}_{2}$ based on the reported electron affinity values in the literature. ${ }^{23,28}$ Based on these alignments, they are expected to form a type-III, broken-gap heterojunction similar to InAs/ $\mathrm{GaSb}$ tetrahedral 3D semiconductors. The large work function difference leads to an accumulation of holes in BP and electrons in $\mathrm{SnSe}_{2}$ near the junction, and an effective $p-i-n$ junction is formed in our devices owing to the presence of the aforementioned interfacial layer (Figure 1i). Away from the junction, it is assumed that the unintentional $p$-type doping is 
such that it puts the Fermi level of p-BP $\left(E_{f p}\right)$ near its valence band edge $\left(E_{v p}\right)$, and the doping in $n$-SnSe $e_{2}$ puts its $E_{f n}$ near its conduction band edge $\left(E_{c n}\right)$. Both layers are thus effectively "degenerately" doped. This junction, made of a heavily doped $p$-region and a heavily doped $n$-region separated by a thin tunneling barrier forms the heart of the Esaki diode, enabling the observation of NDR, as will be discussed in the following text. Though the tunnel barrier in this work is formed unintentionally, thin $\mathrm{BN}$ layers or other suitable barrier materials with improved quality ${ }^{29}$ can be used: which is the focus of our future work.

Figure 2a presents the measured $I_{d}-V_{d s}$ curves at 80 and 300 $\mathrm{K}$ in a linear scale, where the most notable feature is the appearance of NDR, while the junction is positively biased $\left(V_{d s}\right.$ $>0)$. The same data at RT are plotted in a logarithmic scale in Figure 2b (blue circles) to showcase the backward diode behavior: current under reverse bias is higher than that at forward bias, opposite to a typical $p-n$ diode. The simultaneous appearance of NDR and backward-diode behavior is a conclusive fingerprint of interband tunneling transport of electrons. All results presented in this work are obtained by applying a drain voltage $V_{d s}$ on BP (p-type, terminal " $d$ ") with the $\mathrm{SnSe}_{2}$ (n-type, terminal " $s$ ") being grounded. For example, for $V_{d s}>0$ electrons drift through the conduction band of $n$ type $\mathrm{SnSe}_{2}$, tunnel through the barrier into the empty valence band states in $p$-type BP, and drift through this valence band to emerge at the BP ohmic contact (see Figures $1 b$ and $2 e, f$ ). The band alignments of the vdW Esaki diode close to the $\mathrm{BP} / \mathrm{SnSe}_{2}$ interface under various bias conditions are sketched in Figure $2 \mathrm{c}-\mathrm{f}$. At equilibrium, the Fermi level $E_{f}$ lies between $E_{v p}$ and $E_{c n}$ (also see Figure 1i). When a reverse bias voltage $V$ is applied (Figure 2d), a finite energy window is created for electrons to tunnel from the filled valence band states of BP into the empty conduction band states in $\mathrm{SnSe}_{2}$ : the transport is driven by the difference between the hole quasi Fermi level $E_{f p}$ and electron quasi Fermi level $E_{f n}$ that satisfy: $E_{f p}-E_{f n}=q V$, where $q$ is the elementary charge. Increasing the reverse bias simultaneously widens the energy window for tunneling and increases the tunneling probability because of a stronger field, leading to a sharp increase in the tunneling current. This is clearly observed in our devices, the region " $\mathrm{d}$ " in Figure $2 \mathrm{~b}$, and captured accurately with a tunneling transport model as described later. Under a small forward bias $\left(0<q V_{d s}<\Delta E_{n}\right.$, where $\Delta E_{n}=E_{f n}-$ $\left.E_{c n}\right)$, electrons residing in the conduction band of $n-\mathrm{SnSe}_{2}$ are able to tunnel to the empty states in the valence band of $p$-BP through the energy window of $E_{f n}-E_{f p}$ (Figure 2e). This tunneling current reaches its peak when the occupied conduction band states in $\mathrm{SnSe}_{2}$ have a maximal overlap with the unoccupied valence band states in BP. Further increase of the voltage aligns the occupied conduction band states with the forbidden bandgap of BP. Then the tunneling current decreases in spite of a slight increase in tunneling probability induced by a stronger electric field across the barrier. The diode current reaches its minimum (valley) value, which is typically dominated by a combination of phonon assisted tunneling and thermionic currents, ${ }^{37}$ followed by an upturn due to the overriding thermionic emission current in conventional Esaki diodes with high quality junctions. For the device shown in this work, no satisfactory model was found based on the temperature dependence of the post-valley current $(0.3 \mathrm{~V}<$ $V_{d s}<1 \mathrm{~V}$ ), see Figure S2. The metal contacts on BP become Schottky-like at low temperatures; furthermore, the current in this bias window is likely dominated by tunneling into defect states residing in the bandgap of $\mathrm{BP}$ and $\mathrm{SnSe}_{2}$ and via gap states in the tunneling barrier.

A simple model of interband tunneling using the WentzelKramers-Brillouin (WKB) approximation is able to capture all the essential experimental tunneling features for $V_{d s}<0.3 \mathrm{~V}$ quantitatively in these vdW heterojunction Esaki diodes. While the details of the model appear in the Supporting Information (SI), its essence is described briefly here. The device tunneling current is calculated by summing the individual single-particle contribution from all electron states in the $k$-space that are allowed to tunnel: ${ }^{13}$

$$
\begin{aligned}
I_{t}= & q \frac{g_{s} g_{v}}{L_{z}} \sum_{k_{n}} \sum_{k_{p}} v_{z}\left(F_{n}-F_{p}\right) T_{W K B} \delta\left(E_{p}-E_{n}\right) \delta\left(k_{x p}-k_{x n}\right) \delta \\
& \left(k_{y p}-k_{y n}\right)
\end{aligned}
$$

where $g_{s}$ and $g_{v}$ are the spin and valley degeneracy in the bandstructure, $L_{z}$ is the macroscopic device length along the electric field direction $z, v_{z}=\hbar k_{z n} / m_{z n}$ is the band group velocity in the source side, $F_{n}$ and $F_{p}$ are the Fermi-Dirac distribution functions, respectively, in $n-\mathrm{SnSe}_{2}$ and $p$-BP, and $T_{W K B}$ is the WKB tunneling probability. The three Dirac- $\delta$ functions in eq 1 simultaneously ensure energy $(E)$ conservation and in-plane momentum $\left(k_{x}\right.$ and $\left.k_{y}\right)$ conservation. The numerically evaluated tunneling current is shown in Figure $2 \mathrm{~b}$ as a red solid line and is seen to explain the entire tunneling portion of the current: both in reverse and forward bias, before the thermionic/diffusive-part takes over at large forward bias. The model incorporates an effective barrier lowering of $\Delta \phi_{\mathrm{b}}=$ $\left(q \Xi / 4 \pi \epsilon_{r} \epsilon_{0}\right)^{1 / 2}$ where a relative permittivity $\epsilon=6$ is used and $\Xi$ is the electric field across the tunnel barrier. At $300 \mathrm{~K}$, the best fit of the model to the experimental tunneling current is obtained by setting $\Delta E_{p}=E_{v p}-E_{f p}=0.27 \mathrm{eV}, \Delta E_{n}=E_{f n}-E_{c n}$ $=0.07 \mathrm{eV}$, a barrier height $E_{b}=4.7 \mathrm{eV}$, and a tunneling barrier width $t=1.3 \mathrm{~nm}$. The effective doping induced degeneracies and energy scales are reasonable, and the tunnel barrier thickness is in agreement with the physical barrier thickness measured by STEM for the vdW $p+/ i / n+$ heterointerface.

Recognizing that the tunneling probability is exponentially dependent on the barrier height and thickness, a peak tunnel current map is generated as a function of these two parameters in Figure 3. For example, the experimentally observed peak current at RT can be reproduced using a barrier thickness of 2 $\mathrm{nm}$ with a barrier height of $2 \mathrm{eV}$ as well as a barrier thickness of $1.3 \mathrm{~nm}$ with a barrier height of $4.7 \mathrm{eV}$. Considering the effective tunneling area might be smaller than the physical overlap region area $\left(\sim 10 \times 10 \mu \mathrm{m}^{2}\right)$ due to the potential surface contamination during dry transfer, we believe the property of the tunnel barrier between $\mathrm{BP}$ and $\mathrm{SnSe}_{2}$ is most likely spatially distributed and largely falls in the parameter window explored in Figure 3. We note that momentum and energy conservation is invoked in our model with a goal to provide a quantitative estimate of the band alignment between BP and $\mathrm{SnSe}_{2}$. However, the theoretical calculations show that the conduction band minimum of bulk $\mathrm{SnSe}_{2}$ is situated at the $\mathrm{L}$ point in the $k$ space $^{38}$ while the valence band maximum of bulk BP is at the $\mathrm{Z}$ point. ${ }^{39}$ Furthermore, the $\mathrm{BP}$ and $\mathrm{SnSe}_{2}$ flakes stacked in this work most likely have random crystallographic orientations relative to each other, and the interfacial tunnel barrier contains defects that can assist tunneling. All these factors render an accurate tunneling model a daunting task at this stage, thus left to future investigations. We recognize that the necessary 


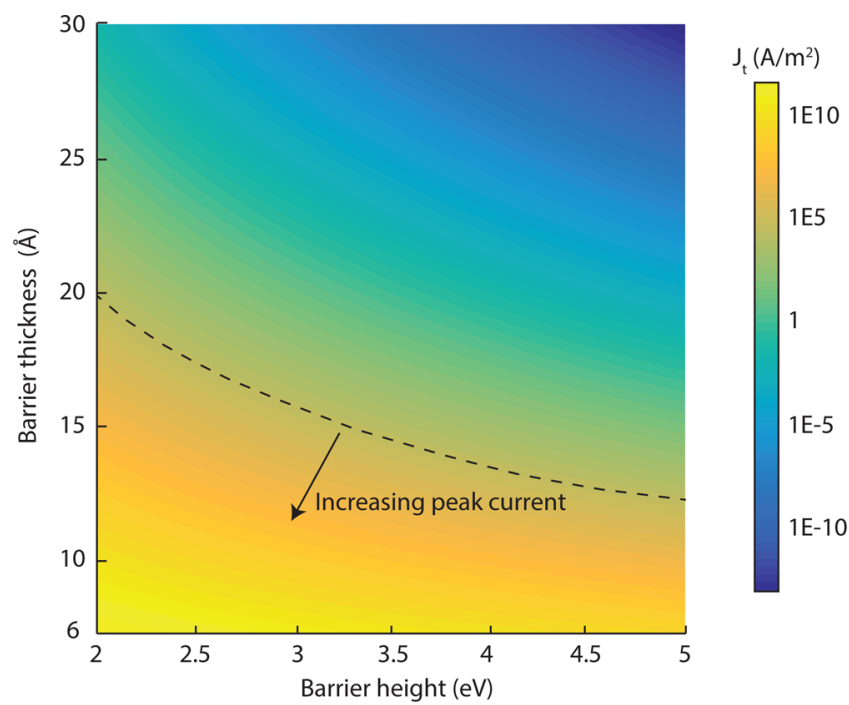

Figure 3. Calculated peak current density in the $\mathrm{BP} / \mathrm{SnSe}_{2}$ Esaki diode mapped over a range of tunnel barrier heights and thicknesses. The dash line marks the measured peak current density shown in Figure 2, which is around $1.6 \times 10^{3} \mathrm{~A} / \mathrm{m}^{2}$. For the modeled $I_{d}-V_{d s}$ curve shown in Figure 2, the following parameters: $E_{b}=4.7 \mathrm{eV}$ and $t=13 \AA$, render an excellent match with the measured $I_{d}-V_{d s}$.

scattering events modify the tunneling probability, which can be lumped in an effective WKB tunneling probability. Therefore, the simplified model presented in this work is sufficient to describe the experimental results well without losing generality.

A number of $\mathrm{BP} / \mathrm{SnSe}_{2} \mathrm{vdW}$ diodes have been fabricated, among which several showed stable NDR behavior under forward bias, while some showed backward diode behavior without NDR. The NDR in those samples is washed out by overwhelmingly high valley currents under forward bias. More information is provided in Supplementary Figures S4 and S5.

The current contribution from tunneling mechanism is expected to have a weak dependence on temperature to the extent of thermal smearing of the Fermi occupation functions. To verify this, we performed temperature-dependent $I_{d}-V_{d s}$ measurements. Figure 4 shows the measured and modeled $I_{d}-$ $V_{d s}$ curves for temperatures ranging from 80 to $300 \mathrm{~K}$. The measured temperature dependence is in excellent agreement with the model, highlighting its predictive capability in spite of the simplicity. A critical feature in the characteristics is the evolution of the peak current $I_{p}$ in the NDR region as a function of temperature: $I_{p}$ increases slightly with decreasing $T$. The Fermi-Dirac tail shortens with decreasing temperature, making the electron occupation probability difference in the $n$ and $p$-sides $F_{n}-F_{p}$ larger, increasing $I_{p}$. This temperature dependence is in stark contrast to thermionic emission, or phonon/trap-assisted tunneling, which typically shows an opposite trend of decreasing current with decreasing temperature. The peak voltage is observed to shift to a higher value at lower temperatures because the ohmic contacts confirmed at RT become Schottky-like at lower temperatures (see Supplementary Figure S2a,b). For the same reason, the reverse bias currents are seen to be slightly lower at low $T$ than that at RT. The inset of Figure 4a shows that the extracted peak-tovalley ratio (PVR) of NDR varies from 2.8 at $80 \mathrm{~K}$ to 1.8 at 300 K. Similar trends are also observed in Esaki diodes made of 3D semiconductors, such as $\mathrm{Ge}$ and $\mathrm{Si}^{1,2}$
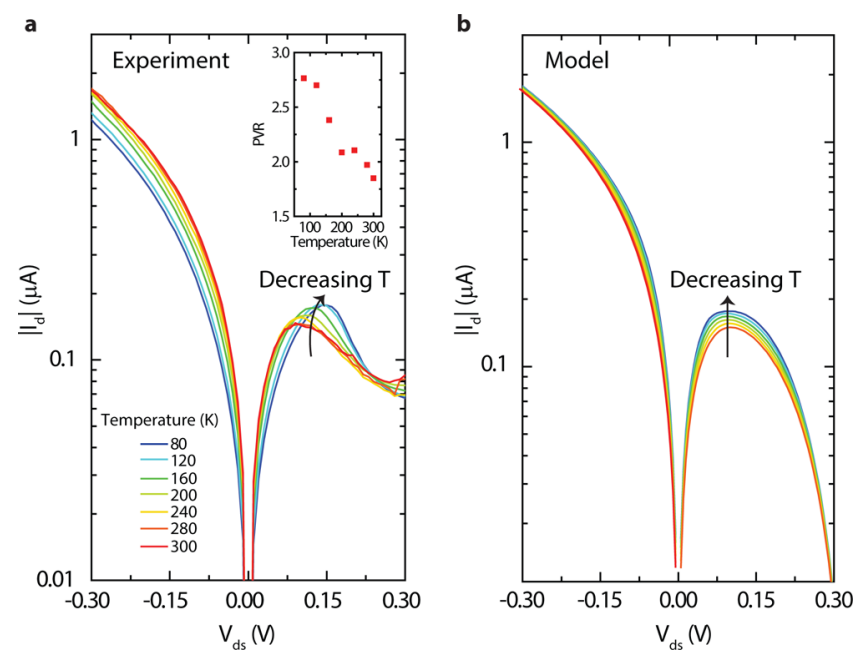

Figure 4. Temperature dependent current-voltage characteristics of the $\mathrm{BP} / \mathrm{SnSe}_{2}$ Esaki diode. (a,b) Experimental and theoretical $I_{d}-V_{d s}$ curves in a temperature range of 80 to $300 \mathrm{~K}$. As temperature decreases, the peak current increases as a result of a tighter FermiDirac distribution of the carriers. The devices suffered from poor ohmic contacts at low temperatures, which shifts the peak voltage to a higher value under the forward bias and lowers the apparent tunneling current under the reverse bias. Inset of (a) shows the measured peak to valley ratio as a function of temperature.

The broken-gap energy band alignment of the $\mathrm{BP} / \mathrm{SnSe}_{2}$ $\mathrm{vdW}$ interface is unusual and of high interest for future applications. The tunneling current model provides the first sign of this band alignment. To conclusively verify the brokengap energy alignment, we explore the photoresponse of the heterojunction under illumination from a $488 \mathrm{~nm}$ laser, as shown in Figure 5. Since the Esaki diode forms a perfect "ohmic" contact near zero voltage, the sign of the photocurrent and photovoltage uniquely correlates with the energy band bending in the heavily doped $p+$ and $n+$ regions near the
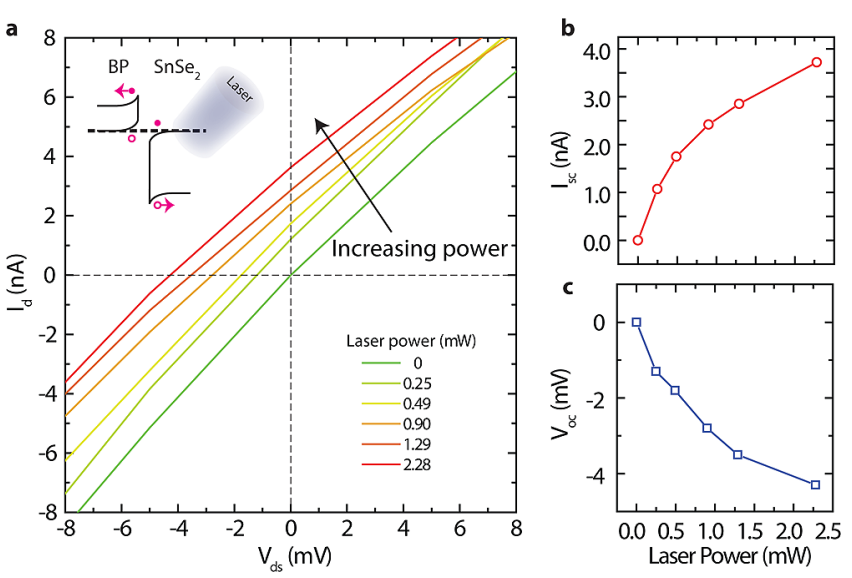

Figure 5. Current-voltage behavior of the $\mathrm{BP} / \mathrm{SnSe}_{2}$ Esaki diode under illumination of a $488 \mathrm{~nm}$ laser. (a) $I_{d}-V_{d s}$ curves near zero bias. Laser power is varied from 0 to $2.28 \mathrm{~mW}$, and the laser beam diameter is about $3 \mu \mathrm{m}$ illuminating the $\mathrm{vdW}$ junction only, excluding the metal/2D regions. The sign of the short-circuit current $I_{s c}$ and the open-circuit voltage $V_{o c}$ is opposite to that of a common $p-n$ junction or solar cell, confirming the band bending in BP (accumulation of holes) and $\mathrm{SnSe}_{2}$ (accumulation of electrons) is thus the type-III or broken band alignment. The inset shows this confirmed band bending. $(\mathrm{b}, \mathrm{c}) I_{s c}$ and $V_{o c}$ as a function of the laser power. 
junction. If the band bending is such that carriers of opposite sign accumulate near the junction as shown in the inset of Figure 5a, the resulting photocurrent will move the Esaki diode $I-V$ curve up into the second quadrant. If the band bending is such that carriers of opposite sign deplete near the junction as a typical $p-n$ diode, the photoresponse $I-V$ will move into the fourth quadrant, like a solar cell. Provided that the Fermi level in $p$-BP $\left(n-\mathrm{SnSe}_{2}\right)$ is very close to its valence (conduction) band edge as argued above, the movement of the $I-V$ curve into the second quadrant in Figure $5 \mathrm{a}$ and the resulting negative open-circuit voltage are signature of a broken-gap alignment. Though it is impossible to rule out the formation of unintentional charged states at the vdW heterointerface in this study, the energy band alignment in $\mathrm{BP} / \mathrm{SnSe}_{2}$ diode is most likely of type-III. The observed Esaki diode behavior and its peculiar photoresponse, combined with the reported properties of the layered materials point strongly toward this conclusion. The detailed discussion on various band alignment possibilities can be found in the SI. With an absorption coefficient of $\sim 5 \times 10^{5} \mathrm{~cm}^{-1}$ of $\mathrm{SnSe}_{2}$ bulk crystals at $488 \mathrm{~nm}$, ${ }^{40}$ we estimate the laser power reaching the vdW heterojunction, and the device responsivity at zero bias to be $\sim 0.24 \mathrm{~mA} / \mathrm{W}$. This value is comparable to those reported in other layered materials. $^{23}$

In conclusion, we have demonstrated an Esaki tunnel diode using vdW heterojunctions with a type-III energy band alignment composed of $\mathrm{BP}$ and $\mathrm{SnSe}_{2}$ for the first time. Our study suggests $2 \mathrm{D}$ crystals as promising candidates for future tunneling based devices. Finally, we point out that Esaki et al. observed NDR in metal-insulator-SnTe junctions, where they realized that the degenerate $p$-type doping of $\mathrm{SnTe}$ coupled with the modulation of the tunneling probability with voltage was primarily responsible for the observed $\mathrm{NDR}^{4}$ In that study, they realize the crucial role played by the fact that the band alignment of the metal and the semiconductor are not "locked" as in epitaxial III-V heterostructures, but can slide with respect to each other because of the presence of the insulating barrier. They call this a new type of tunneling, which indeed is identical to what we see in the $\mathrm{BP} / \mathrm{SnSe}_{2}$ layered semiconductor heterojunction. However, this story has an even older precedent, in Holm and Meissner's experiments in 1930s, where they attributed the resistance measured between two metals to tunneling of electrons through a "void" between them. ${ }^{41}$ In the layered heterojunction materials, the lack of strong interlayer covalent bonds presents exactly such an electronic void, which helps decouple the band alignment and allows the robust NDR observed here. On a more general note, we mention that the robust Esaki-type NDR reported in this work is not entirely new and has been seen over the past half century in various group-IV and III-V semiconductor heterostructures. However, its observation in layered semiconductors that lack out of plane chemical bonds is potentially a starting point for several investigations in the future. Unlike III-V and group-IV semiconductors and their heterostructures, layered semiconductors can be semiconducting, insulating (BN), but also metallic and/or superconducting $\left(\mathrm{NbSe}_{2}\right)$. This means one can create seamless heterostructures of semiconductors with superconductors and insulators without interface traps and investigate the tunneling of particles, be it of single-particle type (in semiconductors) or correlated (in superconductors) between various material families. The fine structures in such tunneling experiments can also serve as the most sensitive tools for the measurement of band alignments, phonon modes, elastic vs inelastic tunneling physics, and a host of potentially unforeseen physics.

Methods. Device Fabrication. The device fabrication starts with the mechanical exfoliation of BP flakes (Smart-element $\mathrm{GmbH}$ ) onto a $285 \mathrm{~nm}$ thick $\mathrm{SiO}_{2}$ covered silicon substrate using scotch tape. The second layered material $\mathrm{SnSe}_{2}$ (2D Semiconductor Inc.) is first exfoliated onto commercially available elastomeric films as stamps. The films (Gel-Fim WF $\mathrm{x} 4$ 6.0mil) were supplied by Gel-Pak. After locating the appropriate thin flakes on the stamp film, the film is turned upside down and aligned with the target BP flake using micromanipulators under an optical microscope. Once the desired alignment is achieved, the stamp is pressed against the substrate and then removed slowly to ensure that the $\mathrm{SnSe}_{2}$ flake remains adhered to the BP flake. This all-dry transfer process avoids liquid contamination and can be completed in a reasonably short amount of time, both of which are critical for fabricating high quality devices. ${ }^{26} \mathrm{Ti} / \mathrm{Au}$ metal contacts (10/ $100 \mathrm{~nm}$ thick) are defined by electron beam lithography and a lift-off process. A Ti/Au stack was deposited on the backside of the $\mathrm{Si}$ substrate to serve as the back-gate.

Photocurrent Measurement. Photocurrents were measured with a Keithley 2636A source meter under optical excitation from a laser of wavelength $488 \mathrm{~nm}$ using an $8 \times$ objective lens with NA of 0.2 . The laser spot size is around $1.22 \lambda / \mathrm{NA} \approx 3$ $\mu \mathrm{m}$, which is much smaller than the size of the heterostructure overlapping area between BP and $\mathrm{SnSe}_{2}\left(\sim 10 \times 10 \mu \mathrm{m}^{2}\right)$.

STEM Specimen Preparation and Imaging. A crosssectional specimen of the device was prepared by using a standard lift-out procedure in a dual-beam FEI Strata 400 focus ion beam (FIB) system, with a final milling at $2 \mathrm{keV}$. After removal from the FIB system, the specimen was directly transferred into an ultrahigh vacuum bake-out system. The specimen was baked in the ultrahigh vacuum chamber for $8 \mathrm{~h}$ at $130{ }^{\circ} \mathrm{C}$ to clean the specimen before loading into the high vacuum of the electron microscope. During this procedure, the specimen experienced an approximately $1 \mathrm{~min}$ long exposure to air. HAADF-STEM images were taken with a Nion UltraSTEM 100 operated at $100 \mathrm{kV}$. The imaging condition was similar to that in ref 42 . The beam convergence angle was 35 $\mathrm{mrad}$, with a probe current of $\sim 70 \mathrm{pA}$. The acquisition time was $32 \mu$ s per pixel.

The EELS spectrum and images were acquired with an energy dispersion of $0.5 \mathrm{eV} /$ channel using a Gatan Quefina dual-EELS Spectrometer. The beam convergence angle was 35 mrad, with a probe current of $\sim 130 \mathrm{pA}$. A linear combination of power laws (LCPL) was used to fit and subtract the background. The EELS false-color composition map was created by integrating the $\mathrm{Sn}-\mathrm{M}_{4,5}$ edge and $\mathrm{P}-\mathrm{L}_{2,3}$ edge. Here we use the $\mathrm{Sn}-\mathrm{M}_{4,5}$ edge since the $\mathrm{Se}-\mathrm{M}_{4,5}$ edge was overwhelmed by a plasma peak on the BP side. All the EELS analysis was done with open-source Cornell Spectrum Imager software. $^{43}$

\section{ASSOCIATED CONTENT}

\section{S Supporting Information}

The Supporting Information is available free of charge on the ACS Publications website at DOI: 10.1021/acs.nanolett.5b01792.

Detailed derivation of tunnel current in $\mathrm{BP} / \mathrm{SnSe}_{2}$ heterostructures, additional electrical and TEM characterizations of the reported device, the reproducibility of 
vdW Esaki diodes with NDR, the observation of backward diode behavior without NDR, and deduction of band alignment from photocurrent measurements (PDF)

\section{AUTHOR INFORMATION}

\section{Corresponding Authors}

*E-mail: ry253@cornell.edu.

*E-mail: grace.xing@cornell.edu.

\section{Author Contributions}

R.Y., D.J., and H.G.X. conceived experiments and conducted device modeling. R.Y. performed device fabrication, electrical and optoelectronic measurements, and data analysis. R.Y., B.S., and S.F. conducted low temperature measurements. V.P. helped on optical measurements. Y.H. took the HAADFSTEM images and conducted EELS spectrum analysis under supervision of D.A.M. S.X., M.L., and N.M. helped with data analysis. R.Y., D.J., and H. G. X. wrote the manuscript. All authors have given approval to the final version of the manuscript.

\section{Notes}

The authors declare no competing financial interest.

\section{ACKNOWLEDGMENTS}

This work was supported in part by NSF CARRER, NSF, AFOSR, and the Center for Low Energy Systems Technology (LEAST), one of the six SRC STARnet Centers, sponsored by MARCO and DARPA. The device fabrication is performed at Cornell Nano-Scale Science and Technology Facility, funded by National Science Foundation (Grant ECCS-0335765). This work made use of the electron microscopy facility of the Cornell Center for Materials Research (CCMR) with support from the National Science Foundation Materials Research Science and Engineering Centers (MRSEC) program (DMR 1120296). We thank Prof. Alan Seabaugh, Dr. Pei Zhao, Malcolm Thomas, and Megan Holtz for useful discussions.

\section{REFERENCES}

(1) Esaki, L. New phenomenon in narrow germanium $\mathrm{p}-\mathrm{n}$ junctions. Phys. Rev. 1958, 109, 603-604.

(2) Esaki, L. Long journey into tunnelling. Rev. Mod. Phys. 1974, 46, 237-244.

(3) Giaever, I. Electron tunneling and superconductivity. Rev. Mod. Phys. 1974, 46, 245-250.

(4) Koelle, D.; Kleiner, R.; Ludwig, F.; Dantsker, E.; Clarke, J. Hightransition-temperature superconducting quantum interference devices. Rev. Mod. Phys. 1999, 71, 631-686.

(5) Bouchiat, V.; Vion, D.; Joyez, P.; Esteve, D.; Devoret, M. H. Quantum coherence with a single Cooper pair. Phys. Scr. 1998, 165, 1998.

(6) Sze, S. M.; Ng, K. K. Physics of semiconductor devices; Wiley: New York, 2006.

(7) Sirtori, C.; et al. Resonant tunneling in quantum cascade lasers. IEEE J. Quantum Electron. 1998, 34, 1722-1729.

(8) Chen, C. J. Theory of scanning tunneling spectroscopy. J. Vac. Sci. Technol., A 1988, 6, 128.

(9) Seabaugh, A. C.; Zhang, Q. Low-voltage tunnel transistors for beyond CMOS logic. Proc. IEEE 2010, 98, 2095-2110.

(10) Zhou, G. et al. Novel gate-recessed vertical InAs/GaSb TFETs with record high $\mathrm{I}_{\text {on }}$ of $180 \mu \mathrm{A} / \mu \mathrm{m}$ at $\mathrm{V}_{\mathrm{DS}}=0.5 \mathrm{~V}$. In Electron Devices Meet. (IEDM), 2012 IEEE Int. 32-36 (IEEE, 2012).

(11) Jena, D. Tunneling transistors based on graphene and 2-D Crystals. Proc. IEEE 2013, 101, 1585-1602.
(12) Li, M.; Esseni, D.; Snider, G.; Jena, D.; Grace Xing, H. Single particle transport in two-dimensional heterojunction interlayer tunneling field effect transistor. J. Appl. Phys. 2014, 115, 074508.

(13) Britnell, L.; et al. Resonant tunnelling and negative differential conductance in graphene transistors. Nat. Commun. 2013, 4, 1794.

(14) Zhao, P., Feenstra, R. M., Gu, G.; Jena, D. SymFET: A proposed symmetric graphene tunneling field effect transistor. In Device Res. Conf. (DRC), 2012 70th Annu. 33-34 (IEEE, 2012).

(15) Britnell, L.; et al. Field-Effect tunneling transistor based on vertical graphene heterostructures. Science 2012, 335, 947-950.

(16) Britnell, L.; et al. Strong light-matter interactions in heterostructures of atomically thin films. Science 2013, 340, 1311-4.

(17) Furchi, M.; et al. Photovoltaic effect in an electrically tunable van der Waals heterojunction. Nano Lett. 2014, 14, 4785-4791.

(18) Hong, X.; et al. Ultrafast charge transfer in atomically thin $\mathrm{MoS}_{2} / \mathrm{WS}_{2}$ heterostructures. Nat. Nanotechnol. 2014, 9, 682-686.

(19) Fang, H.; et al. Strong interlayer coupling in van der Waals heterostructures built from single-layer chalcogenides. Proc. Natl. Acad. Sci. U. S. A. 2014, 111, 6198-202.

(20) Lee, C.-H.; et al. Atomically thin $\mathrm{p}-\mathrm{n}$ junctions with van der Waals heterointerfaces. Nat. Nanotechnol. 2014, 9, 676-681.

(21) Cheng, R.; et al. Electroluminescence and photocurrent generation from atomically sharp $\mathrm{WSe}_{2} / \mathrm{MoS}_{2}$ heterojunction $\mathrm{p}-\mathrm{n}$ diodes. Nano Lett. 2014, 14, 5590-5597.

(22) Roy, T.; et al. Dual-Gated $\mathrm{MoS}_{2} / \mathrm{WSe}_{2}$ van der Waals tunnel diodes and transistors. ACS Nano 2015, 9, 2071-2079.

(23) Deng, Y.; Lou, J.; Xu, X.; Ye, P. D. Black phosphorus/monolayer $\mathrm{MoS}_{2}$ van der Waals heterojunction p-n diode. ACS Nano 2014, 8 , $8292-8299$

(24) Williams, R. H.; Murray, R. B.; Govan, D. W.; Thomas, J. M.; Evans, E. L. Band structure and photoemission studies of $\mathrm{SnS}_{2}$ and $\mathrm{SnSe}_{2}$ I. Experimental. J. Phys. C: Solid State Phys. 1973, 6, 3631.

(25) Li, L.; et al. Black phosphorus field-effect transistors. Nat. Nanotechnol. 2014, 9, 372-7.

(26) Castellanos-Gomez, A.; et al. Deterministic transfer of twodimensional materials by all-dry viscoelastic stamping. 2D Mater. 2014, $1,011002$.

(27) Jamieson, J. C. Crystal structures adopted by black phosphorus at high pressures. Science 1963, 139, 1291-1292.

(28) Robertson, J. Electronic structure of $\mathrm{SnS}_{2}, \mathrm{SnSe}_{2}, \mathrm{CdI}_{2}$ and $\mathrm{PbI}_{2}$. J. Phys. C: Solid State Phys. 1979, 12, 4753.

(29) Cao, Y. et al. Quality heterostructures from two dimensional crystals unstable in air by their assembly in inert atmosphere. arXiv Prepr. arXiv1502.03755 (2015).

(30) Castellanos-Gomez, A.; et al. Isolation and characterization of few-layer black phosphorus. 2D Mater. 2014, 1, 025001.

(31) Wood, J. D.; et al. Effective passivation of exfoliated black phosphorus transistors against ambient degradation. Nano Lett. 2014, 14, 6964-6970.

(32) Su, Y.; Ebrish, M. a.; Olson, E. J.; Koester, S. J. SnSe 2 field-effect transistors with high drive current. Appl. Phys. Lett. 2013, 103, 263104.

(33) Liu, Y.; et al. Two-dimensional mono-elemental semiconductor with electronically inactive defects: the case of phosphorus. Nano Lett. 2014, 14, 6782.

(34) Yan, R; et al. Determination of graphene work function and graphene-insulator-semiconductor band alignment by internal photoemission spectroscopy. Appl. Phys. Lett. 2012, 101, 022105.

(35) Fang, H.; et al. High performance single layered $\mathrm{WSe}_{2} \mathrm{p}$-FET with chemically doped contacts. Nano Lett. 2012, 12, 3788-3792.

(36) Franklin, A. D.; et al. Variability in carbon nanotube transistors: improving device-to-device consistency. ACS Nano 2012, 6, 11091115.

(37) Kane, E. O. Theory of tunneling. J. Appl. Phys. 1961, 32, 83-91.

(38) El-Nahass, M. M. Optical properties of tin diselenide crystals. J. Mater. Sci. 1992, 27, 6597-6604.

(39) Li, L.; et al. Black phosphorus field-effect-transistors. Nat. Nanotechnol. 2014, 9, 372-377. 
(40) Asahina, H.; Moritaournals, A. Band structure and optical properties of black phosphorus. J. Phys. C: Solid State Phys. 1984, 17, 1839.

(41) Holm, R; Meissner, W. Messungen mit Hilfe von flüssigem Helium. Eur. Phys. J. A 1932, 74, 715-735.

(42) Muller, D. A.; et al. Atomic-scale chemical imaging of composition and bonding by aberration-corrected microscopy. Science 2008, 319, 1073-1076.

(43) Cueva, P.; Hovden, R.; Mundy, J. A.; Xin, H. L.; Muller, D. A. Data processing for Atomic Resolution Electron Energy Loss Spectroscopy. Microsc. Microanal. 2012, 18, 667-675. 